\title{
Attitudes Toward Naloxone Prescribing in Clinical Settings: A Qualitative Study of Patients Prescribed High Dose Opioids for Chronic Non-Cancer Pain
}

\author{
Shane R. Mueller, MSW ${ }^{1,2}$, Stephen Koester, $P h D^{2,3}$, Jason M. Glanz, PhD ${ }^{1,4}$, Edward M. Gardner, MD $^{5}$, \\ and Ingrid A. Binswanger, MD, MPH ${ }^{1,6}$
}

\begin{abstract}
'Institute for Health Research, Kaiser Permanente Colorado, Denver, CO, USA; ${ }^{2}$ Department of Health and Behavioral Sciences, University of Colorado Denver, Denver, CO, USA; ${ }^{3}$ Department of Anthropology, University of Colorado Denver, Denver, CO, USA; ${ }^{4}$ Department of Epidemiology, Colorado School of Public Health, Denver, CO, USA; ${ }^{5}$ enver Health and Hospital Authority, Denver, CO, USA; ${ }^{6}$ Division of General Internal Medicine, University of Colorado School of Medicine, Denver, CO, USA.
\end{abstract}

\begin{abstract}
BACKGROUND: Over the last 2 decades, medical providers have increasingly prescribed pharmaceutical opioids for chronic non-cancer pain, while opioid overdose death rates have quadrupled. Naloxone, an opioid antagonist, can be prescribed to patients with chronic pain to reverse an opioid overdose, yet little is known about how patients perceive this emerging practice.

OBJECTIVE: This study assessed the knowledge and attitudes toward naloxone prescribing among non-cancer patients prescribed opioids in primary care.

DESIGN: Qualitative study design using semi-structured interviews.

PARTICIPANTS: Adults $(N=24)$ prescribed high-dose ( $\geq 100$ morphine mg equivalent daily dose) chronic opioid therapy in eight primary care internal medicine, family medicine and HIV practices in three large Colorado health systems.
\end{abstract}

APPROACH: Inductive and deductive methods were used to analyze interview transcripts.

KEY RESULTS: Themes emerged related to knowledge of and benefits, barriers and facilitators to naloxone in primary care. Patients reported receiving limited education about opioid medication risks from providers and limited knowledge of naloxone. When provided with a description of naloxone, patients recognized its ability to reverse overdoses. In addition to pragmatic barriers, such as medication cost, barriers to naloxone acceptance included the perception that overdose risk stems from medication misuse and that providers might infer that they were misusing their opioid medication if they accepted a naloxone prescription, prompting an opioid taper.

Facilitators to the acceptance of naloxone included medical providers' using empowering, non-judgmental communication practices, framing naloxone for use in "worst case scenarios" and providing education and training about opioids and naloxone.

CONCLUSIONS: While patients recognized the utility of naloxone prescribing, we identified important barriers to

Electronic supplementary material The online version of this article (doi:10.1007/s11606-016-3895-8) contains supplementary material, which is available to authorized users.

Received June 3, 2016

Revised September 20, 2016

Accepted September 28, 2016

Published online October 31, 2016 patient acceptance of naloxone prescribing. To improve the naloxone prescribing acceptability in primary care practice, medical providers and health systems may need to enhance patient education, employ empowering, nonjudgmental communication styles and adequately frame discussions about naloxone to address patients' fears.

KEY WORDS: opioids; primary care; naloxone; overdose; qualitative research.

J Gen Intern Med 32(3):277-83

DOI: $10.1007 / \mathrm{s} 11606-016-3895-8$

(C) Society of General Internal Medicine 2016

\section{INTRODUCTION}

Over the last 2 decades, medical providers in family practice, general practice and internal medicine have increased opioid prescribing for chronic non-cancer pain (CNCP), representing nearly half of the opioids dispensed in the US. ${ }^{1,2}$ Alongside an increase in sales, death rates from prescription opioids have quadrupled since 1999. ${ }^{3,4}$ Thus, primary care-based strategies to address the opioid overdose epidemic are needed.

Naloxone is a short-acting opioid antagonist used to counter the effects of an opioid overdose. Recently, the Food and Drug Administration approved intramuscular and intranasal devices for use by non-medical personnel. ${ }^{5,6}$ Federal and professional guidelines, such as the Centers for Disease Control and Prevention's (CDC) Guideline for Prescribing Opioids for Chronic Pain, have endorsed naloxone prescribing for patients at risk of overdose. $^{7-9}$ The education that accompanies naloxone prescriptions can also help patients understand the overdose risks associated with opioid medications. ${ }^{10}$ Large health systems have now begun to promote naloxone "co-prescribing" for patients prescribed opioids for CNCP. ${ }^{11-13}$

Prior research suggests that providers may be reluctant to prescribe naloxone to patients with CNCP because of several logistical and attitudinal barriers. ${ }^{14}$ One important provider concern was that patients with pain may be offended or feel stigmatized by the offer of a naloxone prescription. ${ }^{14}$ However, little is known about whether this concern is justified and how patients perceive overdose education and naloxone 
prescribing in primary care. Our study was designed to understand patients' perspectives on these practices. We also sought to identify barriers to and facilitators of patient acceptance of naloxone. Our goal was to help equip providers with strategies to enhance patient acceptance of naloxone.

\section{METHODS}

\section{Study Design}

Between June 2014 and November 2015, we conducted qualitative interviews of patients prescribed high-dose opioids for CNCP. A qualitative approach was used to elicit participants' personal (emic) perspectives on overdose education and naloxone prescribing, given the minimal available knowledge about patients' views of this emerging practice. ${ }^{15}$ This study was approved by the Colorado Multiple Institutional Review Board and the Kaiser Permanente Colorado Institutional Review Board. We received a Federal Certificate of Confidentiality.

\section{Study Setting}

We recruited patients from eight separate clinics within three health systems: a large network of federally qualified health care centers (Denver Health), a managed care organization (Kaiser Permanente Colorado) and an academic medical center (University of Colorado Hospital). In two systems, takehome naloxone was prescribed to patients who presented to the emergency department for an overdose. In one system, primary care providers received letters identifying patients who could be good candidates for naloxone based on receipt of high-dose [>200 mg morphine equivalent dose (MME)] opioids. During the conduct of the study, Colorado passed legislation that permitted medical professionals to prescribe naloxone to individuals as well as family members, friends or other bystanders in a position to assist an individual experiencing an overdose. ${ }^{16}$ Legislation was also passed permitting outpatient pharmacy naloxone distribution under a standing order. ${ }^{17}$ A standing order is a written instruction issued by a medical practitioner that authorizes pharmacists to distribute naloxone directly to patients without a prescription.

\section{Participants}

Using electronic medical and pharmacy record data, we identified patients who were 21-65 years old and had received three or more opioid prescriptions on three distinct dates within a 90day period with an average daily dose of $100 \mathrm{MME}$ or more. We then excluded patients with a "Do Not Resuscitate" status or cancer diagnosis in the previous 12 months. Among patients identified using electronic health record data, we randomly selected 186 participants, who were recruited using mailed letters in five waves. We conducted additional eligibility screening of those who expressed an interest in participating to determine whether they were still patients at one of our participating health systems and still on high-dose opioid therapy (i.e., had not tapered to a lower dose). Those who provided informed consent and completed an interview received a $\$ 25$ gift card to a local grocery store as compensation.

\section{Methodology}

The interdisciplinary research team, consisting of clinicians, an anthropologist and health services researchers, developed a semi-structured interview guide informed by the findings of prior clinician focus groups, ${ }^{14}$ the Theory of Planned Behavior $^{18}$ and the Health Belief Model ${ }^{19}$ (see sample questions in the Online Appendix). The primary interviewer was a doctoral student with training in qualitative methods (S.M.), who was assisted by a doctoral level anthropologist (S.K.). While the focus of the study was on patient perspectives, two participants felt more comfortable being interviewed with a caregiver. Thus, we allowed caregivers, including family members and close associates, to accompany participants during interviews. Participants (including caregivers) provided informed consent. During the interview, participants were asked to characterize their prior education about the risks of opioid medications, concerns about overdose from their medications, knowledge of and experience with overdose and knowledge about naloxone. Since many participants had limited knowledge about naloxone, the interview guide used the terms "Narcan" (the brand name) and "naloxone" and included a description of naloxone/Narcan. Participants were also asked to reflect on their communication with and trust in their providers and how they would want to receive future information about naloxone and overdose. Participants completed a brief survey of their demographics, opioid medication use and history of drug abuse treatment.

\section{Data Analysis}

Interviews were recorded, transcribed and uploaded into ATLAS.ti software. Using the theoretical models that informed our interview guide content and our prior findings, we created an a priori template of codes. ${ }^{14,20}$ Using this template as a guide, two analysts (S.K. and S.M.) independently coded two transcripts for both manifest and latent content meaning ${ }^{21}$ using ethnographic, iterative methods previously described. ${ }^{14}$ The analysis incorporated both inductive and deductive approaches by assigning predefined codes to text (deductive) and assigning new codes to emergent findings (inductive). ${ }^{22}$ Other research team members (I.B., J.G. and E.G.) applied this code list to an additional two transcripts to refine dimensions of the existing codes and identify new codes. The revised code list was then applied to the additional transcripts. In frequent meetings of the entire research team, the team discussed coding inconsistencies and convergences, identified additional codes emerging from subsequent interviews, refined the coding scheme and ensured consensus, thus improving the validity, consistency and credibility of our findings. ${ }^{23}$ Codes were subsequently categorized into larger groupings, representing themes. Interviews were conducted until the study team agreed that thematic saturation had been reached. ${ }^{24}$ 


\section{RESULTS}

Of 186 randomly sampled patients sent recruitment letters, 60 (32\%) expressed interest in participating, and $51(27 \%)$ met all eligibility criteria. Twenty-four $(13 \%)$ participants (two with their caregivers) completed a semi-structured interview ranging in duration from 35 to $90 \mathrm{~min}$. The remaining eligible participants $(n=26)$ did not schedule or attend their interview appointment. Table 1 shows that participants had a mean age of 53.9 years, were mostly female $(66.6 \%)$ and reported being prescribed opioids on average 11.2 years (median $=8.0$ years).

Below, we provide themes that related to our underlying theoretical models and emerged from the data.

\section{Barriers}

Patient-level barriers that reduced enthusiasm for naloxone are represented in six themes: (1) limited prior education about opioid risks; (2) limited knowledge about naloxone; (3) low perception of overdose risk; (4) fear of exacerbating providers' concerns about opioid misuse; (5) fear of consequences and loss of pain treatment if naloxone is used; and (6) pragmatic barriers.

\section{Limited Prior Education About Risks of Opioid Medications.} Participants reported that they had received little prior overdose education in primary care. When asked about any education that they had received about overdose from their medical provider, participants struggled to recall discussions about the risks of opioid medications. If these discussions had occurred, they took place years previously at the initiation of opioid treatment and had not been revisited in subsequent visits. Participants acquired knowledge about overdose risk from their own prior experiences or those that family members or close associates had experienced.

Table 1 Participant Characteristics $(N=24)$

\begin{tabular}{ll}
\hline \hline Age in years, mean [standard deviation (SD)] & $53.9(7.2)$ \\
Female, no. (\%) & $16(66.6)$ \\
Race/ethnicity, no. (\%) & \\
White, non-Hispanic & $18(75.0)$ \\
African American, non-Hispanic & $4(16.7)$ \\
Hispanic & $2(8.3)$ \\
Education, no. (\%) & $1(4.2)$ \\
Less than high school graduate & $6(25.0)$ \\
High school graduate or GED certificate & $9(37.5)$ \\
Some college & $5(20.8)$ \\
College graduate & $3(12.5)$ \\
Any post-graduate work & $8.0(0.83-44)$ \\
Length of time prescribed opioids in & \\
years, median (range) & $5(20.8)$ \\
Ever received treatment for drug abuse, no. $(\%)$ & \\
Clinic location, no. (\%) & $11(45.8)$ \\
Denver Health & $7(29.2)$ \\
Kaiser Permanente Colorado & $6(25.0)$ \\
\hline University of Colorado Hospital & \\
\hline
\end{tabular}

Respondent: I have a very good friend that had a daughter and her daughter overdosed on medication and she died...It's nothing to play with. It's serious medication. Something not to be played with or used as a recreation drug.

Other sources of information included popular media:

I don't know much about overdose. I don't have any personal experience with it. I don't know anybody who has overdosed except what I've read in the newspapers.

In some cases, patients described past opioid risk behaviors, in part due to lack of knowledge and education:

There's not too much education about it [overdose]... When I first started taking it [the opioid medication], no one told me about OD [overdose] or anything about that. Because I was taking it not [as] prescribed...I was just like when I felt pain I would just take like five or six of them or whatever. Then at the end, I'd run out.

Limited Knowledge About Naloxone. Participants in our study were largely unfamiliar with naloxone.

Interviewer: Have you ever heard of naloxone or Narcan before?

Respondent: Never have.

If there was familiarity with naloxone, it was generally in the context of heroin use rather than opioid use for CNCP.

As much as I know it's [naloxone] for a heroin overdose.

Low Perception of Overdose Risk. Some participants believed that opioids were associated with inherent risks. These participants understood that simply using opioid medications, even as prescribed, could put someone at risk for an overdose and they tended to perceive themselves at risk of an overdose:

Interviewer: So, do you feel like you....are at risk for overdose?

Respondent: I always think there's a risk, yeah...because it's still... a pain medication.

In contrast, another group of participants associated overdose risk with being confused, attempting suicide or "abusing" the medication. These participants dismissed the possibility of experiencing an overdose because they were taking their medications "as prescribed" and only to treat their pain.

I know that people have died [from an overdose]... Yeah, it's serious, but I think also that either they had mixed their pills, you know, and got confused or some 
people really just right out abuse them and was trying to get a high or something and abused them or were seriously trying to kill themselves and did it.

That's the way I see that, but I think if you take them as prescribed, you're OK.

Associating medication abuse with overdose led participants to perceive their overdose risk as negligible. These participants did not see a need for naloxone.

Interviewer: Do you think it would be helpful to have naloxone prescribed to you?

Respondent: No, because I don't over take my medicine. I only take it as prescribed. I've never overabuse...I don't abuse my medication so why would I need it?

In general, a prescription for naloxone was perceived to be beneficial for people who might abuse their medications, but unhelpful for those who use their medications "as prescribed."

You need to find a drug addict who overdoses all the time... it [naloxone] might work for them, but for me, it won't work.

Fear of Exacerbating Providers' Concerns of Misuse. Another barrier to naloxone acceptance was participants' fear of exacerbating medical providers' perceptions of them as "drug abusers." Participants reported that their medical providers were inherently skeptical of their pain and motivations for seeking pain treatment and that they had to continually prove their pain was legitimate.

And so it's not like you go in and you go, 'I'm in pain, can I have some Percocet ${ }^{\mathrm{TM}}$ ?'.... You have to prove to them...that you need the pain relief.

Participants' perceptions that their medical providers did not believe or legitimize their pain contributed to difficult interactions with providers around pain treatment. This struggle to be perceived as an "honest" patient with "legitimate pain" influenced the way that patients interpreted naloxone prescriptions.

If I had not heard what your description [of naloxone] was, I would probably almost be offended or something. I might be like you think I'm abusing them [my medications].

One participant described how she had to battle preconceptions of patients with chronic pain as "addicts" to obtain treatment for her pain:

I think they [previous medical providers]... believe that people with chronic pain are addicts. They just put them in that category. I mean I don't know what it's like for other people but it's just been a battle...I don't need that. I have a life to live.

These concerns were reinforced by poorly communicated opioid policy changes and risk mitigation practices, such as urine toxicology screens.

Interviewer: You talked about also having to do the UA's [urine analyses] for a previous doctor...

Respondent: Oh yeah, you do that at the pain clinic every time...I hated it because I had to move to Kentucky and they did it and I wasn't used to it and it does make you...feel at first like you're...there's something wrong...you're an addict.

Participants were worried that naloxone prescribing in primary care would exacerbate providers' concerns about opioid misuse.

Of course it's [naloxone] going to raise red flags. They're [providers] going to wonder why you need that drug [naloxone]...I mean maybe the pain department would understand that better, but I don't think so.

In the context of increasing use of risk mitigation strategies, intended to limit opioid diversion and misuse, participants interpreted naloxone prescribing as a judgment on the legitimacy of their pain and their motives for seeking pain treatment. One participant, who acknowledged the utility of being prescribed naloxone, demonstrated a reluctance to embrace naloxone because she did not wish to reinforce the stereotype that she was misusing her medication:

Well, I guess you could look at it two ways. You could look at it as well, you know, if [an overdose] should accidentally happen, [naloxone] would be a good thing, or you could think, 'Well, do they think I'm at risk for [misusing my medications]?... or are they doing this more out for my protection?

By accepting naloxone, participants worried that they would unwittingly reinforce medical providers' skepticism of their pain and concern that they were at risk of overdose:

[The] thing about it [accepting naloxone] is that you are giving the signal that you believe that you are going to intentionally overdose because you're already saying I'm going to overdose, I need something for it.

Fear of Reprisals, Such as Loss of Opioid Pain Therapy. Participants expressed concern that accepting naloxone, or using it to reverse an overdose, could negatively influence their provider's perception of them, resulting in reprisals such as the loss of opioid pain therapy. One participant with HIV 
described being rebuked by his medical provider for being honest about taking extra opioids after vomiting some opioid tablets he had taken with his antivirals. This prior experience made him reluctant to disclose potential naloxone use events to his provider:

I was totally honest before and I got screwed...If I agreed to have this [naloxone] in my house and God forbid something happened and I had to use it or someone had to give it to me or whatever, I don't think I'd come back and tell [my doctor] that I had to use it. Just because of the way that everything's been treated...

Participants also reported that a fear of opioid medication tapers negatively influenced their trust in and open communication with their providers. One participant was afraid that her provider was "going to take my [opioid] medicine away and I'm going to be in pain."

Pragmatic Barriers to the Acceptance of Naloxone. Patients identified a number of pragmatic barriers to accepting naloxone. These barriers included concerns about the cost of naloxone, possible adverse events and drug interactions, fear of the immediate physical effects of naloxone, how long naloxone would last before expiring and the absence of a caregiver to administer naloxone. These and other pragmatic barriers are reported in Table 2 with illustrative quotes.

\section{Facilitators}

Potential facilitators to patient acceptance of naloxone included (1) recognition of the utility of naloxone to prevent overdose death, (2) providers who engaged in empowering and non-judgmental communication practices, (3) naloxone

Table 2 Pragmatic Barriers to Naloxone Acceptance

\begin{tabular}{|c|c|}
\hline Barriers & Illustrative quotation \\
\hline Cost & $\begin{array}{l}\text { "A lot of people can't afford medicine, } \\
\text { you know, it depends on how much } \\
\text { this stuff costs..." }\end{array}$ \\
\hline Expiration & $\begin{array}{l}\text { "What's the shelf life on something } \\
\text { like [naloxone]?" }\end{array}$ \\
\hline $\begin{array}{l}\text { Presence of someone to } \\
\text { administer naloxone }\end{array}$ & $\begin{array}{l}\text { "See, and that [administering naloxone] } \\
\text { would be hard because I live alone" }\end{array}$ \\
\hline $\begin{array}{l}\text { Ability to identify an } \\
\text { overdose }\end{array}$ & $\begin{array}{l}\text { "I'm uncomfortable with... self- } \\
\text { diagnosing [an overdose]" }\end{array}$ \\
\hline $\begin{array}{l}\text { Ability to administer } \\
\text { naloxone correctly }\end{array}$ & $\begin{array}{l}\text { "Do you put it [naloxone] in the arm, the } \\
\text { leg, the fatty tissue, the stomach?" }\end{array}$ \\
\hline Fear of inducing pain & $\begin{array}{l}\text { "But also at the administration of this } \\
\text { medication, wouldn't my pain level shoot } \\
\text { up?" }\end{array}$ \\
\hline Fear of withdrawal effects & $\begin{array}{l}\text { "Would I ever want to take it [naloxone]? } \\
\text { It sounds like its effects are nasty enough } \\
\text { that (laughing), I don't know" }\end{array}$ \\
\hline Fear of drug interactions & "I'm scared of drug interactions" \\
\hline Adverse events of the drug & $\begin{array}{l}\text { "[I would need to know] if there's any } \\
\text { danger of like being allergic to it...stuff } \\
\text { like that" }\end{array}$ \\
\hline
\end{tabular}

framing and (4) training and education for patients and caregivers who might administer naloxone.

Recognition of the Utility of Naloxone. Participants who believed that taking opioid medications was inherently risky viewed naloxone as a valuable tool that could be lifesaving in the event of an overdose.

Interviewer: How helpful do you think it would be to have Narcan at home in case you overdosed?

Respondent: It would be very helpful...just in the event that it may happen, it'd be nice to know I have that preventive...medicine there for me.

Empowering, Non-Judgmental Communication Practices. Participants suggested that providers' approaches to prescribing naloxone were paramount to patients being receptive to naloxone.

They should know how to approach a person who might benefit from it [naloxone] in such a way that it's not offensive or judgmental.

Participants characterized empowering communication practices as listening and being responsive to their needs, having a non-judgmental attitude, recognizing and giving legitimacy to the patient's subjective experience of pain and involving them as active participants in their pain treatment plans. One participant emphasized the value of listening to patients without transmitting personal values and views onto the patient:

Non-judgmental, listen and respond and don't put your values or views on that person...take out your personal feelings and listen to the person.

How Naloxone is Framed. Participants raised several ways of framing naloxone that could enhance its acceptability. Framing naloxone as a medication only to be used in a "worst case scenario" resonated with patients who did not consider themselves at risk for an overdose as it communicates the "riskiness" of the medications without judgment. Comparisons between naloxone and other safety measures that are useful in unexpected situations, such as seat belts and fire extinguishers, resonated with participants.

It's like a seat belt. You don't plan on getting in an accident but if you do it's good to have the seat belt. I can see the fire extinguisher analogy...it doesn't mean you're going to go set a fire, but it's [naloxone] there just in case so it could save lives.

These approaches to framing were acceptable to participants because they highlighted the risks of the medications and value of naloxone, while reducing concerns about the perceived association between naloxone and medication "abuse." 
Adequate Training and Education. In addition to receiving a prescription for naloxone, participants described the importance of being educated about the risks of overdose: "Education is definitely the key." Another participant described the importance of training on when and how to use naloxone:

I would want to have a class or a long discussion with a doctor so that it was recognized when it [naloxone] was appropriate and how to use it.

Education and training would be beneficial to address many of the pragmatic barriers cited in Table 2, such as the ability to identify an overdose and administer naloxone correctly.

\section{DISCUSSION}

Our study explored patients' perspectives on naloxone prescribing for patients on chronic opioid therapy in primary care settings. Our findings highlight gaps in prior education about overdose risk among patients prescribed high-dose opioid therapy. Findings suggest that providers should continuously educate patients about the risks throughout the course of treatment. When provided with a description of naloxone, patients recognized its utility and ability to reverse potentially fatal overdoses. While participants understood the utility of naloxone, they generally did not perceive themselves to be at risk for an overdose. They equated overdose risk with misuse of opioid medications, which in turn limited their perceived need for naloxone and its acceptability. The thought that requesting naloxone would prompt their provider to consider tapering them off their pain medication represented a significant barrier to naloxone acceptance. These concerns have implications for how providers communicate the inherent risks of opioid medications and consequences of naloxone use with their patients.

Prior research in community-based overdose education and naloxone distribution programs (not based in health clinics) has suggested that participants were unfamiliar with naloxone, but strongly in favor of receiving naloxone. ${ }^{25-28}$ These programs typically serve people who use illicit pharmaceutical opioids and heroin and who may be more likely to administer naloxone to peers than participants in our study. Unlike patients in primary care settings, participants in community-based programs do not have to fear losing access to opioids by accepting a naloxone prescription. A study of US veterans prescribed opioids for opioid use disorder treatment and/or CNCP reported that participants were generally unaware of naloxone, but were interested in receiving a naloxone prescription. ${ }^{29}$ Due to low perceived overdose risk, participants in our study believed that a prescription for naloxone would be beneficial for other patients, but less beneficial for themselves.
The recently published CDC Guideline for Prescribing Opioids for Chronic Pain encourage medical providers to use urine drug testing to assess patients for use of illicit drugs, monitor their patients using prescription drug monitoring programs and adhere to opioid dose thresholds. ${ }^{9}$ As a result, providers may feel compelled to adhere to these guidelines. In light of our findings, these risk mitigation practices could alienate patients and hinder open dialogue about risk behavior and naloxone acceptance.

To improve our understanding of naloxone uptake in primary care, future qualitative studies could explore the perceptions of caregivers and patients who decline naloxone prescriptions. We sampled patients from a single state but diverse health care systems. We found few appreciable differences in patient perspectives across sites. Future quantitative studies could sample a more nationally representative sample and measure the prevalence and relative importance we identified. Additional studies should then assess whether addressing the barriers we identified would increase naloxone uptake.

Several barriers identified by participants in our study correspond with previously identified provider barriers, ${ }^{14}$ such as the prohibitive cost of naloxone and potentially stigmatizing aspects of naloxone. While providers expressed concern that prescribing naloxone may be considered stigmatizing, ${ }^{14}$ patient participants suggested that naloxone could be prescribed using a patient-centered approach that is empowering and non-judgmental. Naloxone could be framed for use in a "worst case scenario," as "fire extinguisher" or as a "seat belt" to affirm providers' concerns about their patients' safety and potentially increase patients' acceptance of naloxone. Different approaches to framing could be tested in future research. Future research should evaluate whether the universal prescribing of naloxone would result in greater acceptance among CNCP patients and ultimately reduce mortality. Overall, we conclude that despite low perceived overdose risks, naloxone was acceptable to patients on high-dose chronic opioid therapy and that their concerns about naloxone could be addressed through enhanced communication with providers.

Corresponding Author: Shane R. Mueller, MSW; Institute for Health Research, Kaiser Permanente Colorado, 10065 E. Harvard Ave., Suite 300, Denver, CO 80231, USA (e-mail: Shane.Mueller@kp.org).

\section{Compliance with Ethical Standards:}

Funders: Research reported in this publication was supported by the National Institute On Drug Abuse of the National Institutes of Health under Award Number R34DA035952. The content is solely the responsibility of the authors and does not necessarily represent the official views of the National Institutes of Health.

Prior Presentations: Preliminary data from earlier portions of this study were presented at the 38th Annual SGIM meeting in Toronto, Canada, and the 77th Annual CPDD meeting in Phoenix, AZ.

Conflict of Interest: The authors declare that they do not have a conflict of interest. 


\section{REFERENCES}

1. Boudreau D, Von Korff M, Rutter CM, Saunders K, Ray GT, Sullivan MD, et al. Trends in long-term opioid therapy for chronic non-cancer pain. Pharmacoepidemiol Drug Saf. 2009;18(12):1166-75.

2. Levy B, Paulozzi L, Mack KA, Jones CM. Trends in opioid analgesicprescribing rates by specialty, US, 2007-2012. Am J Prev Med. 2015;49(3):409-13.

3. Frenk SM, Porter KS, Paulozzi LJ. Prescription opioid analgesic use among adults: United States, 1999-2012. NCHS Data Brief. 2015;(189): 1-8.

4. Compton WM, Jones CM, Baldwin GT. Relationship between nonmedical prescription-opioid use and heroin use. N Engl J Med. 2016;374(2): 154-63.

5. Kaleo. Evzio ${ }^{\mathrm{TM}}$ prescribing information. Available at: http://evzio.com/ pdfs/Evzio\%20PI.PDF. Accessed September 23, 2016.

6. Adapt Pharma I. Narcan (naloxone hydrochloride) nasal spray package insert. Available at: http://www.accessdata.fda.gov/drugsatfda_docs/label/2015/208411lbl.pdf. Accessed September 23, 2016.

7. Substance Abuse and Mental Health Services Administration. SAMHSA opioid overdose prevention toolkit. HHS publication no. (SMA) 13-4742. Rockville, MD. 2013.

8. Food and Drug Administration, Centers for Disease Control and Prevention, National Institute on Drug Abuse. Role of naloxone in opioid overdose fatality prevention: A public workshop. Silver Spring, MD. 2012.

9. Dowell D, Haegerich TM, Chou R. CDC guideline for prescribing opioids for chronic pain-United States, 2016. MMWR Recomm Rep. 2016;65(1):1-49.

10. Walley AY, Xuan Z, Hackman HH, Guinn E, Doe-Simkins M, SorensenAlawad A, et al. Opioid overdose rates and implementation of overdose education and nasal naloxone distribution in Massachusetts: Interrupted time series analysis. BMJ. 2013;346.

11. Bennett AS, Elliott L, Golub A. Veterans' health and opioid safetycontexts, risks, and outreach implications. Fed Pract. 2015;32:4-7.

12. Oliva EM, Nevedal A, Lewis ET, McCaa MD, Cochran MF, Konicki PE, et al. Patient perspectives on an opioid overdose education and naloxone distribution program in the US Department of Veterans Affairs. Subst Abus. 2016;37(1):118-26.

13. Coffin PO, Behar E, Rowe C, Santos G-M, Coffa D, Bald M, et al Nonrandomized intervention study of naloxone coprescription for primary care patients receiving long-term opioid therapy for painnaloxone coprescribing in primary care clinics. Ann Intern Med. 2016;165(4):245-52.

14. Binswanger IA, Koester S, Mueller SR, Gardner EM, Goddard K, Glanz JM. Overdose education and naloxone for patients prescribed opioids in primary care: a qualitative study of primary care staff. $J$ Gen Intern Med. 2015;30(12):1837-44.
15. Denzin NK, Lincoln YS. The sage handbook of qualitative research: Sage; 2011.

16. Colo. Rev. Stat. § 18-1-711. 2012.

17. Colo. SB15-053. 2015.

18. Montaño DE, Kasprzyk D. Theory of reasoned action, theory of planned behavior, and the integrated behavioral model, chapter 4 . In: Glanz K, Rimer BK, Lewis FM, eds. Health behavior and health education: theory, research, and practice. 4th ed. San Francisco: Wiley; 2008.

19. Champion VL, Skinner CS. The health belief model, chapter 3. In: Glanz K, Rimer BK, Lewis FM, eds. Health behavior and health education: theory, research, and practice. 4th ed. San Francisco: Wiley; 2008.

20. Fereday J, Muir-Cochrane E. Demonstrating rigor using thematic analysis: a hybrid approach of inductive and deductive coding and theme development. Int J Qual Methods. 2006;5(1):1-11

21. Cho JY, Lee E-H. Reducing confusion about grounded theory and qualitative content analysis: similarities and differences. Qual Rep. 2014;19(32): 1 .

22. LeCompte MD, Schensul JJ. Analyzing and interpreting ethnographic data. Walnut Creek, CA: Rowman Altamira; 1999.

23. Barbour RS. Checklists for improving rigour in qualitative research: a case of the tail wagging the dog? BMJ. 2001;322(7294):1115-7.

24. Creswell Jw. Research design: qualitative, quantitative, and mixed methods approaches. Thousand Oaks: SAGE Publications; 2014.

25. Lagu T, Anderson BJ, Stein M. Overdoses among friends: drug users are willing to administer naloxone to others. J Subst Abuse Treat. 2006;30(2):129-33.

26. Seal K, Downing M, Kral A, Singleton-Banks S, Hammond J-P, Lorvick $\mathbf{J}$, et al. Attitudes about prescribing take-home naloxone to injection drug users for the management of heroin overdose: a survey of streetrecruited injectors in the San Francisco bay area. J Urban Health. 2003;80(2):291-301.

27. Strang J, Powis B, Best D, Vingoe L, Griffiths P, Taylor C, et al Preventing opiate overdose fatalities with take-home naloxone: prelaunch study of possible impact and acceptability. Addiction. 1999;94(2): 199-204

28. Kerr D, Dietze P, Kelly AM, Jolley D. Attitudes of Australian heroin users to peer distribution of naloxone for heroin overdose: perspectives on intranasal administration. J Urban Health. 2008;85(3):352-60.

29. Tiffany E, Wilder CM, Miller SC, Winhusen T. Knowledge of and interest in opioid overdose education and naloxone distribution among us veterans on chronic opioids for addiction or pain. Drugs: Educ Prev Polic. 2015;23(4):322-7. 